\title{
Can a nanoparticle that mimics Salmonella effectively combat tumor chemotherapy resistance?
}

\author{
"...engineering of this semisynthetic Salmonella nanoparticle mimic \\ introduces a new platform technology that has the capacity to \\ be applied to various chemotherapeutic drugs to overcome MDR \\ in tumors."
}

First draft submitted: 3 January 2017; Accepted for publication: 9 February 2017; Published online: 21 March 2017

Keywords: cancer $\bullet$ drug delivery $\bullet$ effector proteins $\bullet$ multidrug resistance $\bullet$ nanoparticles - Salmonella • therapeutic adjuvant

Cancer is a group of conditions in which cells acquire a constellation of traits that in the course of carcinogenesis, enables them to survive, achieve sustained chronic proliferation and disseminate. To attain such unremitting subsistence, the tumor cells take advantage of complex cell biological networks that include, but are not limited to: dysregulation of growthpromoting signaling pathways; attenuation of proliferative negative-feedback mechanisms; evasion of growth suppressors; blunting of cell attachment; and circumventing the immune system. Notably, these traits are further cultivated by rampant genetic instability as highlighted by the generation of random mutations and chromosomal rearrangement.

In the USA, cancer is the second leading cause of death - second only to cardiovascular disease. Sadly, and quite profoundly, this complex disease touches millions of lives, and continues to be an immense burden for public health systems and economies world-wide. Putting this into perspective, for the year 2016, the American Cancer Society has projected a total of $1,685,210$ new cancer cases (the equivalent of 4600 new cancer diagnoses/day) [1], and 595,690 of cancer deaths (corresponding to 1600 deaths/day).

\section{Current challenges in cancer treatment}

It is undeniable that enormous strides have been made by clinicians and biomedical researchers in the pursuit of combating neoplastic disease, and such successes have and will continue to advance diagnostic and therapeutic development. Current therapeutic approaches tailored to the molecular determinants that drive tumor growth, have resulted in improvement in treatment outcomes of various types of cancer. For example, HER2, a receptor that drives and activates proliferation/survival pathways such as MAPK and PI3K/Akt, has been the target of multiple adjuvant drugs used in breast and gastric neoplasias. However, anti-HER2 monoclonal antibodies that are currently on the market have only yielded moderate improvements in clinical outcomes [2], and are documented to be associated with severe cardiotoxic events [2]. Additional dysregulated oncogenic signaling pathways, such as antiapoptotic signals, tumor-driven angiogenesis and increased immune evasion, have been the focus of targeted cancer therapies with mixed grades of success. Therefore, any lack of therapeutic success can likely be broadly explained by the complex nature of the disease, its heterogeneous presentation and the fundamental ability of cancer cells to constantly evolve, adapt and switch dependency on different oncogenic signaling pathways. Nevertheless, the desirability of targeted therapies lies in their ability to arrest tumor growth and induce regression, as observed in a molecularly defined cohort

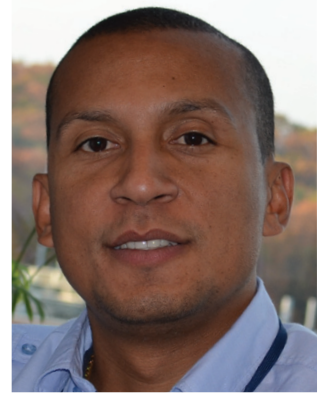

Regino Mercado-

Lubo

Department of Microbiology \& Physiological Systems, University of Massachusetts Medical School, 368

Plantation Street, Worcester, MA 01655, USA

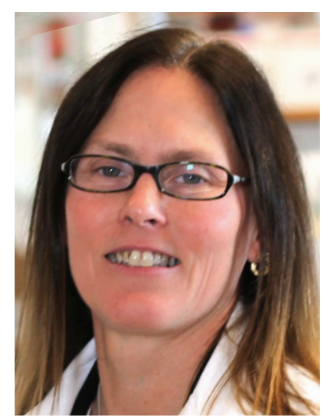

Beth A McCormick Author for correspondence: Department of Microbiology \& Physiological Systems, University of Massachusetts Medical School, 368 Plantation Street, Worcester, MA 01655, USA Tel.: +1 5088566048 Fax: +1 5088563355 Beth.McCormick@ umassmed.edu

Future $\because \because$ Medicine part of 
of patients. However, if used alone, such treatment regimens often fail to kill tumor cells, which notably underscores a critical need for chemotherapeutic drugs in the treatment of cancer patients.

Among the different types of cancer therapies available today (e.g., surgery, radiotherapy, immunotherapy and targeted therapies), chemotherapy continues to be one of the first lines of treatment (and in some cases, the only feasible option). In instances like advanced or metastasized breast cancer, chemotherapy regimens typically include drugs within the taxane and anthracycline families, along with targeted therapy directed against the estrogen receptor, the progesterone receptor, or HER2. However, the effectiveness of such cytotoxic drugs is often restricted by two key factors: resistance to chemotherapeutic agents; and the devastating side effects triggered by them.

\section{"While advances have been made in utilizing AuNPs as a delivery system for genetic material and small molecules, the carriage of functional proteins on nanoparticles with retention or enhanced activity has remained largely unchartered primarily because of the complexities surrounding protein recognition and structure retention. To bypass this limitation, we engineered surface ligands for direct conjugation of SipA to the AuNPs."}

Patients undergoing chemotherapy also face significant side effects that adversely impact their quality of life. Chemotherapy-induced gastrointestinal complications, reduced blood cell counts, infections and fever, electrolyte disorders, malnutrition, peripheral neuropathy and premature menopause in women are common among patients being treated for cancer [3], with younger patients showing greater psychological morbidity [3]. These side effects often debilitate patients and are an unfortunate limiting step in cancer management. Not surprisingly, chemotherapy-induced side effects are considered to be one of the principal burdens faced by both patients and clinicians. While the large majority of cancer-related hospitalizations are precipitated by the disease itself, approximately $30 \%$ are triggered by adverse effects from the treatment [4]. Recent studies have also suggested that the chemotherapy-related side effects may be more common than what is reported by large clinical trials, and lead to more patient suffering and healthcare expenditures than previously estimated [4]. Moreover, these untoward effects not only become a threat to the life of the patients, but also limit the doses that can be administered, ultimately preventing the use of higher doses that could compensate for the limited response of conventional chemotherapy.
Over the past two decades, information garnered from cell biology research has strengthened the understanding of how malignant cells survive toxic insults and become resistant to antineoplastics. Though our understanding of the molecular biology of cancer continues to mature, no successful therapy against drug resistance has been achieved to date. In part, this is likely because cancer cells thrive in a toxic environment, largely owing to their ability to exploit an arsenal of molecular mechanisms that include an increase in drug efflux mechanisms, mutations of drugs targets, DNA damage repair processes, activation of alternative signaling pathways and evasion of cell death cascades. Increased drug efflux, also known as multidrug resistance (MDR), is the principal mechanism by which cancer cells develop resistance to chemotherapic drugs. Indeed, several cell membrane transporters of the large ATP-binding cassette protein family have been associated with the MDR phenotype, preventing the effectiveness of commonly used cancer drugs [5].

\section{P-glycoprotein \& its role in MDR}

Resistance to chemotherapy medications, such as anthracycline may emerge from the action of a variety of membrane transport proteins that promote the extrusion of cytotoxic drugs. The best described is P-glycoprotein (P-gp), a $170-\mathrm{kDa}$ adenosine triphosphate (ATP)-dependent multispecific drug transporter. Reports linking overexpression of the MDR1 gene and P-gp to adverse treatment outcome in many cancers has implicated this MDR phenotype as an important biologic target for pharmacologic modulation [6]. P-gp has been associated with adverse outcomes in a variety of solid tumors that include breast, colon, bladder and ovarian cancer [7]. Since its discovery in 1979, numerous modulators of P-gp have been developed; however, none has been successfully translated into the clinic. P-gp is, therefore, recognized as a major barrier to the bioavailability of cytotoxic drugs, and as a consequence, resistance to chemotherapy remains an obstacle to the successful treatment of human cancer.

To date, however, there have only been a handful of strategies, which have been tested or are proposed to reverse drug resistance. More recent strategies have targeted the apoptosis pathway, as well as incorporating the use of hammerhead ribozymes against the MDR1 gene and MDR1-targeted anti-sense oligonucleotides [8]. Thus, despite more than 20 years of effort, a large number of clinical trials with P-gp-modulating agents have been conducted (so far with poor success), as these drugs were vastly ineffective or only effective at toxic doses. Hence, it appears that current approaches for treating cancer are limited, in part, by the inability of drugs to either affect the poorly vascularized regions 
of tumors or to the development of resistance to the chemotherapeutic drug.

\section{Bacteria as cancer therapeutic}

The concept of applying bacteria as a cancer therapeutic has been considered as a feasible option for more than 150 years. In the late 1800s, observations were made by W Busch and F Fehleisen, which associated bacterial infections with tumor regression in cancer patients [9]. William B Coley came to similar conclusions formed from his observations that a fraction of cancer patients who developed postoperative bacterial infections went into remission and were cured of their tumors [10]. Although the mechanisms behind these observations were not well appreciated, scientists at the time knew about the immunostimulatory properties of bacteria. Furthermore, it has been known for nearly 60 years that anaerobic bacteria can selectively grow in tumors, underscoring the fact that such microbes have the potential to overcome many of the delivery barriers, such as impaired circulation and extensive necrosis, found in tumors that hinder conventional chemotherapeutics [11]. Once inside tumors, these bacteria can also effectively activate cells such as macrophages and dendritic cells [12].

Recent advances have fueled a resurgence of interest in genetically modified bacteria as drug delivery vehicles and/or tumoricidal agents [11]. Several classes of bacteria including Listeria, Clostridia and Salmonella have been tested as potential therapies for cancer, with varying degrees of success [11]. Indeed, novel approaches to developing tumor-therapeutic bacteria have recently been documented. Sasaki et al., for example, found that the anaerobic bacterium Bifidobacterium longum could selectively grow in hypoxic regions of solid tumors [13]. Additionally, Vogelstein et al. created a strain of Clostridium novyi depleted of its lethal toxin and showed that intravenous administration of $C$. novyi spores germinated within the avascular regions of tumors in mice and destroyed surrounding viable tumor cells [14]. Presumably, these bacteria cause tumor cell death largely as a result of outcompeting cancer cells for nutrients. These examples serve to illustrate an intriguing history and useful guidance for the treatment of cancer using live microorganisms. However, numerous challenges such as the safety profile and efficacy in larger cohorts of patients remain as obstacles to clinical development in the application of such bacteria as soldiers of chemotherapeutic agents.

The foodborne pathogen Salmonella enterica serovar Typhimurium ( $S$. Typhimurium) has also been used as a therapy, and as a delivery vehicle for cancer therapeutics. This facultative anaerobe, can survive in both oxygenated and hypoxic conditions, and engage in directed motility toward tumor sites, enticed by the high concentrations of nutrients available within the tumor [15]. Capitalizing on these innate properties, a nonpathogenic strain of $S$. Typhimurium was developed (VNP2009), which lacks important metabolic genes (purI, msbB and $x y l$ ) but has been shown to migrate and accumulate in tumor sites more at a rate 1000-times higher compared with that of healthy tissues [16]. Once it reaches tumors, $S$. Typhimurium is thought to out compete cancer cells for essential nutrients. Although this strain was able to retard tumor growth and prolong survival in mice, it was not successful at slowing tumor growth in the clinic [17]. $S$. Typhimurium was also found to cause the lysis of tumor cells by injecting cytotoxic peptides into the target cells and facilitating a nonspecific immune reaction [17]. Thus, while $S$. Typhimurium can localize to tumors, the biggest shortcoming of this organism as a potential therapeutic is its inability to disperse throughout the tumor.

“

"...in vivo proof of concept studies confirmed that the Salmonella nanoparticle mimic, when used in combination with a common cancer chemotherapeutic (e.g, doxorubicin) causes tumor regression in a subcutaneous model of murine colon cancer, as well as in a patient-derived xenograft mouse model of breast cancer."

Nevertheless, $S$. Typhimurium remains a promising delivery vehicle because of its tumor specificity, and recent advances have further kept alive the hope that Salmonella might one day be developed as a clinically useful anticancer agent. In a recent study published in Nature, Din and colleagues introduced a novel strategy that utilizes $S$. Typhimurium as a potential drug delivery platform. Unique to this method, these researchers engineered a self-limiting strain harnessed with a synchronized lysis circuit that allows the organism to release, in an oscillatory manner, its genetically encoded (toxic) cargo [18]. This synchronized lysis circuit technology was achieved by inserting a quorum-sensing lysis system such that at the end of every lytic cycle, a small number of surviving bacteria reseed the growing population, perpetuating pulsatile delivery cycles. While this technology is lauded as a new path in the field of targeted drug delivery systems, the authors did not report bacterial growth ranges in vivo, raising concerns related to systemic toxicity associated with higher bacterial counts. Although $S$. Typhimurium strains were injected intratumorally, the likelihood of bacteremia remains high. This would facilitate a sustained oscillatory infection of the liver, spleen and bone mar- 
row, which might eventually become toxic for healthy tissues colonized by these strains.

\section{Salmonella effector protein as treatment for cancer}

We have taken a new approach in the use of bacteria as a treatment agent for cancer by capitalizing on an unexpected resource - host-targeting proteins produced by pathogenic bacteria. Salmonella enterica serovar Typhimurium is a Gram-negative bacterial pathogen that causes a self-limiting gastroenteritis. Infection by $S$. Typhimurium is mediated by an array of effector proteins that are delivered sequentially and temporally into targeted host cells through a needle-like apparatus, the Type Three Secretion System (T3SS). These effectors then hijack the structural, immunological and life cycle processes of the host cell [19]. T3SS effector proteins are therefore often multifunctional proteins with several overlapping properties that orchestrate specific host cell responses. Salmonella pathogenicity likely evolved through the possession of Pathogenicity Islands (SPI). While at least seven separate SPIs have been identified, SPI-1 and SPI-2 encode a T3SS to deliver effectors that promote pathogenesis and bacterial survival [20]. One of these, Salmonella invasion protein A (SipA), is a dual function effector protein that plays roles in both actin polymerization [21] and induction of inflammatory responses [22].

\section{"The SipA-nanobug was designed to surmount many of the key challenges commonly associated with traditional or even unconventional chemotherapeutic strategies with enhanced pharmacokinetic and biodistribution proficiencies."}

Since Salmonella has evolved to interface with numerous biochemical pathways of epithelial cells, it seemed reasonable that $S$. Typhimurium might also have evolved mechanisms that interact/interfere with MDR transporters that complicate drug treatment. Therefore, we examined where the two paths of targeting the MDR phenotype, specifically P-gp, and Salmonella's inherent antitumor properties cross in regard to cancer therapeutics and host-tumor/pathogen interactions. We discovered that colonization of human colon cancer cell lines (that overexpress P-gp) by wild-type $S$. Typhimurium leads to a profound functional decrease and loss of protein expression in the MDR protein transporter, P-gp. Of note, this is the first observation to link a microorganism that is targeted to tumors with the regulation of MDR transporters.

More recently, our work has uncovered that the $S$. Typhimurium T3SS effector protein, SipA, is respon- sible for the downregulation of the MDR transporter P-gp. In fact, this protein interacts with a broad spectrum of tumors (colon, breast, bladder and B-cell lymphoma), inhibiting the cellular mechanisms that allow cancer cells to develop resistance to chemotherapeutic drugs. In previous contributions, we have revealed that in Salmonella-infected epithelial cells P-gp expression is downregulated without a corresponding decrease in P-gp mRNA. This observation supports a mechanism of P-gp protein cleavage from the cell membrane rather than the regulation of gene expression, and is consistent with prior studies using human T-lymphoblastoid CEM cells, which were the first to describe that P-gp downregulating can occur through a process involving protein degradation [23]. The ability of the $S$. Typhimurium effector, SipA, to modulate P-gp depends on its ability to activate caspase-3 (CASP3) [24], and further supports our observation that SipA is an inducer of apoptosis.

We, therefore, began to consider whether SipA, itself, could be used as an effective P-gp modulator (and without the undesired side effects). We first showed that purified SipA not only improved the cytotoxic activity of known chemotherapeutic drugs (e.g., doxorubicin and vinblastine), which are also P-gp substrates, but also increased the intracellular retention of these drugs. Second, we built a Salmonella nanoparticle mimic made by fusing a $15 \mathrm{~nm}$ gold nanoparticle with a SipA corona (SipA-AuNP) to be applied as an effective therapeutic adjuvant, as a method to increase cancer cell sensitivity to chemotherapeutics. To create this novel drug/therapeutic protein-delivery system, we used gold nanoparticles (AuNP) as a scaffold. AuNPs have been used in therapy as colloidal pharmaceuticals with a long history of intra-articular application for treatment of chronic arthritis, and display the following key properties tailored for delivery vehicles: the gold core is essentially inert; AuNPs are $<100 \mathrm{~nm}$ in size, which enhances their permeation and retention effect, thereby allowing these particles to efficiently extravasate into interstitial spaces. The end result is a much higher concentration of payload therapeutic at the tumor site relative to the same administered dosage of free drug [25]; nanoparticles as drug delivery systems have been shown to circumvent P-gp-mediated MDR by reducing MDR1 gene expression [26]; nanoparticles can be fabricated in highly divergent ways to provide various desirable delivery modes, such as stabilizing conjugated proteins [27], DNA [28] and drugs [29]. They also can promote long systemic circulation lifetime, along with a higher tumor targeting ability, intracellular thiol (e.g., glutathione), controllable payload release and excellent solubility qualities. 
While advances have been made in utilizing AuNPs as a delivery system for genetic material and small molecules, the carriage of functional proteins on nanoparticles with retention or enhanced activity has remained largely unchartered primarily because of the complexities surrounding protein recognition and structure retention. To bypass this limitation, we engineered surface ligands for direct conjugation of SipA to the AuNPs. Selected ligands containing biocompatible oligo(ethylene glycol) spacers between SipA and the nanoparticle reduce nonspecific interactions, as well as provide additional degrees of freedom and polyvalency for enhancing SipAs activities. This method has yielded conjugates that are able to prevent this protein from dissociation or aggregation, and which preserve the conformation/structure of SipA.

Using this strategy, the SipA-AuNPs significantly increased the stability of surface bound SipA proteins, and reduced P-gp expression across numerous cancer cells at a SipA dose nearly 500 times lower than free unbound SipA. In addition, in vivo proof of concept studies confirmed that the Salmonella nanoparticle mimic, when used in combination with a common cancer chemotherapeutic (e.g, doxorubicin) causes tumor regression in a subcutaneous model of murine colon cancer, as well as in a patient-derived xenograft mouse model of breast cancer. Significantly, in these models it is notable that the efficacy of the chemotherapeutic drug, doxorubicin, was profoundly increased, as the dose of the agent required to reach tumor reduction was lowered between two- to ten-fold compared with the dose conventionally used in the field. This effect is most likely due to the $70 \%$ reduction of P-gp expression observed in these tumors [30].

The SipA-nanobug was designed to surmount many of the key challenges commonly associated with traditional or even unconventional chemotherapeutic strategies with enhanced pharmacokinetic and biodistribution proficiencies. Nonetheless, we acknowledge that the development of drug resistance in cancer cells is

\section{References}

1 Siegel RL, Miller KD, Jemal A. Cancer statistics, 2016. CA Cancer J. Clin. 66(1), 7-30 (2016).

2 Onitilo AA, Engel JM, Stankowski RV. Cardiovascular toxicity associated with adjuvant trastuzumab therapy: prevalence, patient characteristics, and risk factors. Ther. Adv. Drug Saf. 5(4), 154-166 (2014).

3 Kayl AE, Meyers CA. Side-effects of chemotherapy and quality of life in ovarian and breast cancer patients. Curr. Opin. Obstet. Gynecol. 18(1), 24-28 (2006).

4 Hassett MJ, O'malley AJ, Pakes JR, Newhouse JP, Earle CC. Frequency and cost of chemotherapy-related serious adverse a rather complex and multifactorial process. That is, the molecular mechanisms activated to protect cancer cells against toxic compounds and allow them to thrive during their clonal expansion certainly extend beyond a single mechanism of drug resistance. There is also the additional concern that because ATP-binding cassette transporters are a large family of highly conserved proteins, that one transporter can compensate if another is blocked. Regardless, the core of our drug technology is uniquely designed to combat drug resistance which should improve the effectiveness of the treatment. Moreover, given that Salmonella has coevolved with the human host for nearly 2 million years and thus may exploit host machinery not accessible by other drug-based methods, it is not unreasonable to envisage that SipA may afford unique advantages with respect to previously developed small molecules that target MDR. Overall, engineering of this semisynthetic Salmonella nanoparticle mimic introduces a new platform technology that has the capacity to be applied to various chemotherapeutic drugs to overcome MDR in tumors.

\section{Financial \& competing interests disclosure}

This work was supported by NIH grants DK56754 and DK33506, The Worcester Foundation and by a UMCCTS Pilot Project Program award (CTSA grant no. UL1TR000161) awarded to BA McCormick. The authors have no other relevant affiliations or financial involvement with any organization or entity with a financial interest in or financial conflict with the subject matter or materials discussed in the manuscript apart from those disclosed.

No writing assistance was utilized in the production of this manuscript.

\section{Open access}

This work is licensed under the Attribution-NonCommercialNoDerivatives 4.0 Unported License. To view a copy of this license, visit http://creativecommons.org/licenses/by-nc$n d / 4.0 /$

effects in a population sample of women with breast cancer. J. Natl Cancer Inst. 98 (16), 1108-1117 (2006).

5 Szakacs G, Paterson JK, Ludwig JA, Booth-Genthe C, Gottesman MM. Targeting multidrug resistance in cancer. Nat. Rev. Drug Discov. 5(3), 219-234 (2006).

6 Krishna R, Mayer LD. Modulation of P-glycoprotein (PGP) mediated multidrug resistance (MDR) using chemosensitizers: recent advances in the design of selective MDR modulators. Curr. Med. Chem. Anticancer Agents 1(2), 163-174 (2001).

7 Holohan C, Van Schaeybroeck S, Longley DB, Johnston PG. Cancer drug resistance: an evolving paradigm. Nat. Rev. Cancer 13(10), 714-726 (2013). 
8 Fojo T, Bates $S$. Strategies for reversing drug resistance. Oncogene 22(47), 7512-7523 (2003).

9 Oelschlaeger TA. Bacteria as tumor therapeutics? Bioeng. Bugs 1(2), 146-147 (2010).

10 Mccarthy EF. The toxins of William B Coley and the treatment of bone and soft-tissue sarcomas. Iowa Orthop. J. 26, 154-158 (2006).

11 Wall DM, Srikanth CV, Mccormick BA. Targeting tumors with Salmonella Typhimurium - potential for therapy. Oncotarget 1(8), 721-728 (2010).

12 Felgner S, Kocijancic D, Frahm M, Weiss S. Bacteria in cancer therapy: renaissance of an old concept. Int. J. Microbiol. 2016, 8451728 (2016).

13 Sasaki T, Fujimori M, Hamaji Y et al. Genetically engineered Bifidobacterium longum for tumor-targeting enzyme-prodrug therapy of autochthonous mammary tumors in rats. Cancer Sci. 97(7), 649-657 (2006).

14 Dang LH, Bettegowda C, Huso DL, Kinzler KW, Vogelstein B. Combination bacteriolytic therapy for the treatment of experimental tumors. Proc. Natl Acad. Sci. USA 98(26), 15155-15160 (2001).

15 Hoffman RM. Tumor-targeting amino acid auxotrophic Salmonella typhimurium. Amino Acids 37(3), 509-521 (2009).

16 Low KB, Ittensohn M, Luo X et al. Construction of VNP20009: a novel, genetically stable antibiotic-sensitive strain of tumor-targeting Salmonella for parenteral administration in humans. Methods Mol. Med. 90, 47-60 (2004).

17 Toso JF, Gill VJ, Hwu P et al. Phase I study of the intravenous administration of attenuated Salmonella typhimurium to patients with metastatic melanoma. J. Clin. Oncol. 20(1), 142-152 (2002).

18 Din MO, Danino T, Prindle A et al. Synchronized cycles of bacterial lysis for in vivo delivery. Nature 536(7614), 81-85 (2016).

19 Galan JE. Molecular and cellular bases of Salmonella entry into host cells. Curr. Top. Microbiol. Immunol. 209, 43-60 (1996).
20 Hueck CJ. Type III protein secretion systems in bacterial pathogens of animals and plants. Microbiol. Mol. Biol. Rev. 62(2), 379-433 (1998).

21 Zhou D, Mooseker MS, Galan JE. An invasion-associated Salmonella protein modulates the actin-bundling activity of plastin. Proc. Natl Acad. Sci. USA 96(18), 10176-10181 (1999).

22 Lee CA, Silva M, Siber AM, Kelly AJ, Galyov E, Mccormick BA. A secreted Salmonella protein induces a proinflammatory response in epithelial cells, which promotes neutrophil migration. Proc. Natl Acad. Sci. USA 97(22), 12283-12288 (2000).

23 Mantovani I, Cappellini A, Tazzari PL, Papa V, Cocco L, Martelli AM. Caspase-dependent cleavage of $170-\mathrm{kDa}$ P-glycoprotein during apoptosis of human T-lymphoblastoid CEM cells. J. Cell. Physiol. 207(3), 836-844 (2006).

24 Srikanth CV, Wall DM, Maldonado-Contreras A et al. Salmonella pathogenesis and processing of secreted effectors by caspase-3. Science 330 (6002), 390-393 (2010).

25 Wang M, Thanou M. Targeting nanoparticles to cancer. Pharmacol. Res. 62(2), 90-99 (2010).

26 Davis ME, Chen ZG, Shin DM. Nanoparticle therapeutics: an emerging treatment modality for cancer. Nat. Rev. Drug. Discov. 7(9), 771-782 (2008).

27 Hong R, Fischer NO, Verma A, Goodman CM, Emrick T, Rotello VM. Control of protein structure and function through surface recognition by tailored nanoparticle scaffolds. J. Am. Chem. Soc. 126(3), 739-743 (2004).

28 Han G, Martin CT, Rotello VM. Stability of gold nanoparticle-bound DNA toward biological, physical, and chemical agents. Chem. Biol. Drug. Des. 67(1), 78-82 (2006).

29 Rana S, Bajaj A, Mout R, Rotello VM. Monolayer coated gold nanoparticles for delivery applications. Adv. Drug Deliv. Rev. 64(2), 200-216 (2012).

30 Mercado-Lubo R, Zhang Y, Zhao L et al. A Salmonella nanoparticle mimic overcomes multidrug resistance in tumours. Nat. Commun. 7, 12225 (2016). 